\title{
Vereinigte Medizinische Gesellschaft in Kiew, Sektion für
}

Sitzung vom 6. Februar 1929

Vorsitzender : Prof. M. Lewitsky.

1. Priv.-Doz. A. Wassütínsky demonstriert a) einen Kranken, bei dem er während der Extraktion der Katarakt mit Konjunktivalbrücken-lappen die basaie Iridektomie statt einer gewöhnlichen gemacht hat. Am dritten Tage nach der Operation entstand Hyphaema, das gleich verschwand. Enderfolg gut.

b) Fall basaler Iridektomie bei Glaukom. Nach der Operation rasche Augendruckverminderung. Durch gemachtes Iriskolobom beim Ophthal-moskopieren sieht man den Linsenrand, was ein Beweis dafür ist, daß die Iridektomie an der Iriswurzel gemacht wurde. W. hat überhaupt bei Glaukom viermal basale Iridektomie gemacht und ist der Ansicht, daß die basale Iridektomie bei Glaukom überhaupt weniger Gefahr als die gewöhnliche darbietet.

Aussprache: Lewitsky, Korenjewitsch, Poljak.

A. Cholina: Über die Augenpoliklinik des Allukrainischen L·istituts für Hygiene und Erziehung. Die Augenpoliklinik stellt sich folgendc Auf-gaben : 1. den konstitutionellen Bau des Auges und 2. einzelne Mo-mente der Außenwelt in ihrer Wirkung auf das Auge zu erlernen. Die weitere Aufgabe besteht in der Zusammenstellung dieser beiden Mo-mente, um eine gewisse Korrelation zwischen ihnen zu finden und Bedingungen herauszufinden, die maximale Arbeitsleistung bei geringstem Energieverbrauch schaffen könnten. Auf Grund zahlreicher Beobach-tungen, (es sind mehr als 6000 Kinder untersucht worden), hat die Poliklinik Profile für Kinder von 8 bis 16 Jahren ausgearbeitet, die auf eine große Bedeutung der Refraktion in dieser Frage hinweisen. Die Poliklinik leistet eine große Arbeit, zwecks Erlernung der Beleuchtung, Lehrbücher u. s. w. in der Schule. Die Poliklinik hat ihre Methode zur Begutachtung dieser Momente, sowie graphisch sie darzustellen, ausgearbeitet. Es sind viele Beobachtungen über die Wirkung der Errnüdung auf die Akkommodation, Muskelgleichgewicht und Sehschärfe des Kinderauges am Ende des Schultages durchgeführt worden.

Alle Zusammenfassungen sind in der Schrift : ,Auf dem Wege zum neuen Menschen” veröffentlicht worden.

Aussprache: Lewitsky, Wassütinsky, Korenjewitsch.

Sitzung am 20. Februar ıç2ç. A. Rumjanzewa. 1. Fall ton Retinitis haemorragica unbekannter Ätiologie.

2. Bemerkens $\Lambda$ verter Fall von Keratitis neuroparalytica.

Prof. M. Lewitsky demonstriert einen Fall von ausgeprägtem . Lipodermoid eonjunctivae.

O. Miroschnitschenko. Zur Kasuistik infektiöser Augenerkrankungen.

Vereinigte Medizinische Gesellschaft in Kievv.

311

M. bespricht fünf bemerkenswerte Fälle infektiöser Augenerkrankungen, die ihr im Laufe letzter Zeit zur Beobachtung standen: Der erste Fall betrifft ein kleines Mädchen, bei dem nach der Pockenimpfung nach einer Woche an den Lidrändern beider Augen typische pustulöse Pockengeschwüre mit Keratitis enstanden. Wahrscheinlich hat sich das Mädchen selbst 
vakziniert. Nach der Behandlung verschwanden an den Lidrändern die Geschwüre, auf der Hornhaut blieb eine leichte Trubung. Im zweiten Falle war eine schwere Orbitalphlegmone, die nach Erysipelas faciei entstand. Diese Affektion endete mit Applanatio corneae und Erscheinungen sekundären Glaukoms.

Zwei weitere Fälle - Anthrax palpebrarum. In einem Falle wurde das Oberlid in Form Pustula malignum, im zweiten beide Lider eines Auges in Form Anthraxödem betroffen. Bakteriologisch sind Milzbrandbazillen nachgewiesen worden. Die Behandlung bestand in spezifischer Serumtherapie.

Der letzte Fall betrifft Diphtherie der Konjunktiva. Die rechtzeitige und energische Therapie blieb erfolglos und der Prozeß endete mit Bulbusatrophie.

Aussprache: Danilewsky und Mironenko weisen darauf hin, daß die sogenannte ,,russische Behandlung" des Milzbrandes: Subkutane Injek-tion der 5\% Karbollösung in manchen Fallen ziemlich gute Resultate leisten kann.

Lewitsky und Wassütinsky betonen, daß man bei Anthrax diebesten Resultate nur durch konservative Therapie und spezifische Serumtherapie erzielen kann.

Sitzung vom 6. März IÇ2Ç..

(Gemeinsam mit der Sektion für Phtysiatrie).

Vorsitzender: Prof. M. Lewitsky.

1. N. Chramelow, Zur Frage über das Eindringen der tuberkulösen

Bazillen durch den unbeschädigten Konjunktivalsack (vorläufige Mit-

teilung). Um die Durchgangsfähigkeit der tuberkulösen Bazillen fest-

zustellen, wurden Versuche an 8 Meerschweinchen aufgestellt. Beob-

achtungsdauer 2-6 Monate. Die Dosis der einfachen Einträufelung

ins Meerschweinchenauge betrug 0,0005 mg. /. Bowen-Vallêe.

Endschlüsse: a) Die unbeschädigte Konjunktive läßt sehr leicht die Tuberkelbazillen durch.

Eine kleine Zahl von Bazillen geht die Konjunktive durch, ohne darauf primäre Affektion

hervorzurufen.

Eine große Zahl von Bazillen ruft ein typisches tuberkulöses Granulom auf der Konjunktive der

Lider hervor.

A ussprache: Ginsburg: Etwas sonderbar ist, daß alles beim Verfasser zu gut vonstatten ging.

Vossius und Lagrange, die sich auch mit dieser Frage beschäftigten, haben ganz andere Resultate erzielt. Das Experiment soil fortgesetzt werden, aber mit anderen Versuchstieren, weil es

möglich ist, daß alles durch die große Empfindlichkeit der Meerschweinchen-konjunktive erklärt werden kann.

Epstein: Der Vortrag wirft ein neues Licht auf einige Seiten der

312 Vereinigte Medizinische Gesellschaft in Kiew,

Phtysiogenese bei Leuten, aber mit einigen Grundsätzen des Vortrages kann ich nicht

einverstanden sein. Freilich ist es zweifellos, daß Kon-junktive als eine Pforte für tuberkulöse

Infektion eine gewisse Rolle spielen kann.

Tscherkassky: Bei der Auswahl des Experimentaltieres ware es nötig, ein solches Tier zu finden, das seiner Widerstandsfähigkeit nach dem Menschen gleicht. Dazu eignen sich ganz gut die weißen Mäuse.

Angenitsky: Die Endresultate, die der Verfasser erhielt, bestätigen sich auf dem menschlichen

Materiale nicht. 
Lewitsky: Diese Arbeit revidiert die Frage der Augentuberkulose. Nach dem Vortrag geht die Infektion vom Auge aus, aber gewöhnlich kommt sie endogen. Die Versuche müssen nochmals wiederholt werden.

Waschetko: Die Versuche wurden speziell mit Meerschweinchen auf-gestellt, weil letztere eine große Empfindlichkeit zur Tuberkulöse be-sitzen. Sie zeigen, daß eine Möglichkeit vom Eindringen der Infektion von der Konjunktiva aus besteht.

Sobkewitsch: Wenn die tuberkulösen Bazillen durch irgend welche Scbleimhaut hineindringen, so werden die letzteren (Mund, Darm, Kon-junktive) sehr selten selbst betroffen; am meisten erkranken andere Organe.

Gents : Die Versuche sind deswegen interessant, $\lambda$ veil sie beweisen, daß die exogenen tuberkulösen Affektionen nicht zu selten vorkommen können.

2. /. Ginsburg: Fall beiderseitigen Koloboms der Sehnervenpapille.

A ussprache: Poljak meint, daß dieses Kolobom nicht ganz typisch sei. Es ist die Möglichkeit nicht ausgeschlossen, daß die Veränderungen um die Papule herum durch Myopie erklärt werden können.

O. Kaganowa demonstriert: a) Fall von Orbitalangiome. b) Fall $\tau$ on Orbitalsarkom. G.Poljak: Embolie der Zentralarterie. Rotarmeesoldat 22 Jahre. Vor 8 Tagen, um 2 Uhr nachmittags fühlte Patient nach einer raschen Bewegung plötzlieh, daß er auf beiden Augen vollständig blind geworden ist. Dieser Zustand dauerte eine kurze Zeit, nachdem er mit dem rechten Auge anfing zu sehen, während auf dem linken das Sehen nicht zu-rückkam. Befund. R. A. leichte Blässe der Papule, weiter O. B. L. A. klassisches Bild einer Zentralarterieembolie. Vis. o. d. $=0,7$, O. S. $=0$. Therapie resultatlos. Herz ohne Befund. Harn ohne Abweichungen von der Norm. WaR ++. Der Fall ist durch seine Äthiologie interessant, O. Trofimenko: Fall von Konjunktivitis Parineaud.

J.K.Korenj $\beta$ witsch demonstiiert zwei Fälle mit eigenartiger Verwun- $<$ dung der Augen. G. D. Poljak. 